\title{
MEMS AIR-MICROFLUIDIC LAB-ON-A-CHIP SENSOR FOR PERSONAL MONITORING OF AIRBORNE PARTICULATE MATTER
}

\author{
D. Fahimi ${ }^{1}$, O. Mahdavipour ${ }^{1}$, T. Cados ${ }^{2}$, T. Kirchstetter, ${ }^{2}$ P. Solomon ${ }^{3}$, L. Gundel ${ }^{2}$, R. M. White ${ }^{4}$, N. Fukushima ${ }^{5}$, \\ H. Nagai ${ }^{5}$, M. Saitoh ${ }^{5}$, and I. Paprotny ${ }^{{ }^{*}}$ \\ ${ }^{1}$ University of Illinois, Chicago, IL, USA \\ ${ }^{2}$ Lawrence Berkeley National Laboratory, Berkeley, CA, USA \\ ${ }^{3}$ U.S. Environmental Protection Agency, Las Vegas, NV, USA \\ ${ }^{4}$ University of California, Berkeley, CA, USA \\ ${ }^{5}$ Kanomax Inc., Osaka, Japan
}

\begin{abstract}
We present the design, fabrication and experimental results for a novel air-microfluidic lab-on-a-chip sensor, which measures the mass concentration of airborne particulate matter (PM), such as tobacco smoke or diesel exhaust. The sensor detects PM having aerodynamic diameter (AD) of less than $2.5 \mu \mathrm{m}$, (PM2.5) directly by measuring the deposited PM on a mass-sensing resonator, at flowrates two orders of magnitude lower than the closest comparable devices. We report on an improved sensor design, new mask-less dispenser-printed SU-8-based bonding process, and recorded sensitivity of our newly-developed design.
\end{abstract}

\section{INTRODUCTION}

Particulate matter (PM), a mixture of small solid particles floating in the air, is an important type of air pollutant. The US EPA categorizes PM by size into coarse (A.D $>10 \mu \mathrm{m}$ ) and fine (A.D < $2.5 \mu \mathrm{m})$ particles. According to the EPA there are serious health hazards associated with fine particles. They are able to penetrate deep inside the human respiratory system and can cause adverse health effects such as increased respiratory symptoms, aggravated asthma, and cardiovascular problems leading to premature deaths in people with heart or lung diseases.

In this work we present a MEMS air-microfluidics lab-on-achip sensor for personal monitoring of PM2.5. A preliminary version of the sensor was reported in [1-2]. In contrast with other miniature PM sensors (such as. [3]), the device presented in this work uses a microfabricated film-bulk acoustic resonator (FBAR) to directly measure the mass of the deposited PM. In this work we present a newly optimized design of the virtual impactor (VI) and microchannels for balancing flowrates through minor and major channels, the development of a new mask-less adhesive wafer bonding technique using dispenser printing of SU-8, as well as characterization of the VI using a custom opto-gravimetric method. Data are also shown demonstrating sensor response to incense particles. The working flowrate is set to $6 \mathrm{~mL} / \mathrm{min}$ and experimental results show the sensitivity of the sensor to be approximately $5 \mathrm{~Hz}$ $\times \mathrm{m}^{3} / \mu \mathrm{g} \times \mathrm{min}$, with a limit of detection (LOD) of $1 \mu \mathrm{g} / \mathrm{m}^{3}$.

\section{Device operation}

A conceptual block diagram and an image of the MEMS PM2.5 sensor are shown on Figs. 1 and 2, respectively. The sensor consists of three main components fabricated on three wafers (quartz-siliconquartz) bonded together. In the air-microfluidic circuit, PM particles are separated by the aerodynamic diameter using a virtual impactor (Fig. 2-a). PM2.5 and smaller are transported through the major flow channels while the larger particles are exhausted from the sensor through the minor flow channel. PM2.5 is then deposited by a thermophoretic precipitator (Fig. 2-b) on a mass sensitive FBAR. A change in the FBAR's resonant frequency occurs as the mass loading increases, and the rate of this frequency change corresponds to the particle concentration in the sampled air volume.
Thermophoretic precipitation is driven by a microfabricated heater suspended just below the capping quartz wafer of the microfluidic circuit. Forward-looking Infrared (FLIR) analysis (Fig. 2-c) shows the temperature distribution (around $100{ }^{\circ} \mathrm{C}$ ) of the heater while dissipating $50 \mathrm{~mW}$ of power, which is typical for the thermophoretic precipitator in our designs.

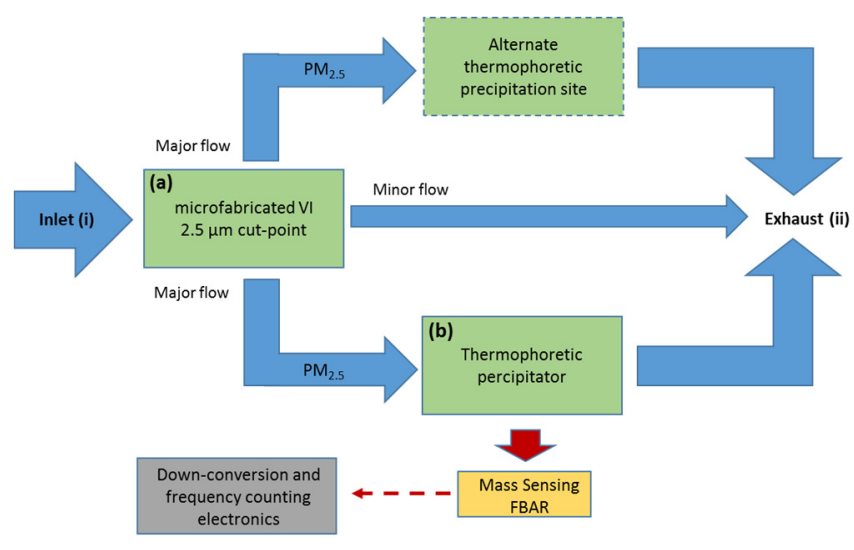

Fig. 1. Conceptual functional diagram describing the operation of the MEMS PM2.5 sensor.

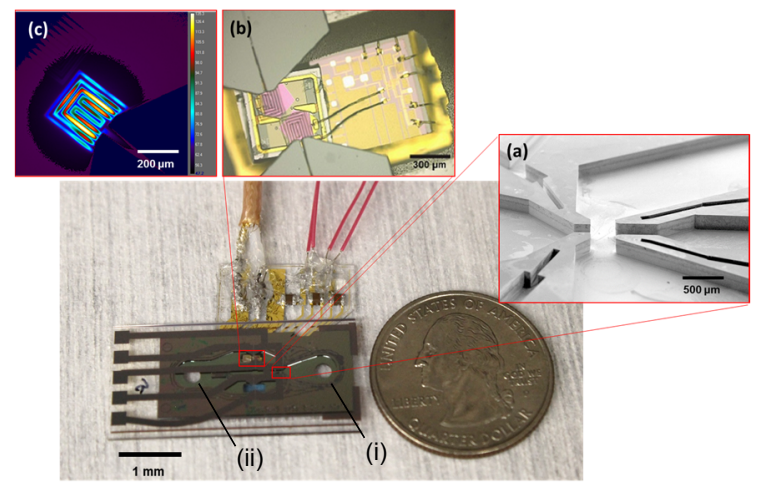

Fig. 2. Air-microfluidic MEMS PM2.5 Sensor. The sensor consists of a microfabricated virtual impactor (a), and a thermophoretic precipitator and mass sensing FBAR (b). FLIR analysis of the active heater dissipating $50 \mathrm{~mW}$ is shown in (c). Air inlett (i) and outlet (ii) is annotated in the lower pane.

\section{FABRICATION}

The sensor is fabricated using a stack of three wafers. A CAD drawing in Fig. 3 schematically displays all the wafers. The fabrication process for the new sensor is shown on Fig. 4. It consists of separately fabricated middle and top wafers components, sub- 
dicing, and subsequent bonding of the two wafer die. A novel adhesive (SU-8 3025) bonding method was used to bond released microfabricated heaters (top wafer) with the etched channels of the middle wafer.

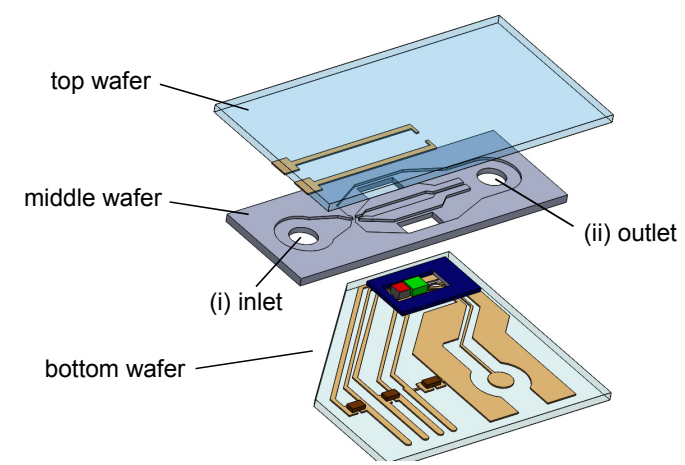

Fig. 3. CAD drawing of all three wafers of the PM2.5 sensor.

The bottom quartz wafer with gold electrodes contains the FBAR and its CMOS driver, providing an oscillating signal at the resonant frequency of the FBAR. The process is finished by attaching the bottom wafer to the stack of middle and top wafer.
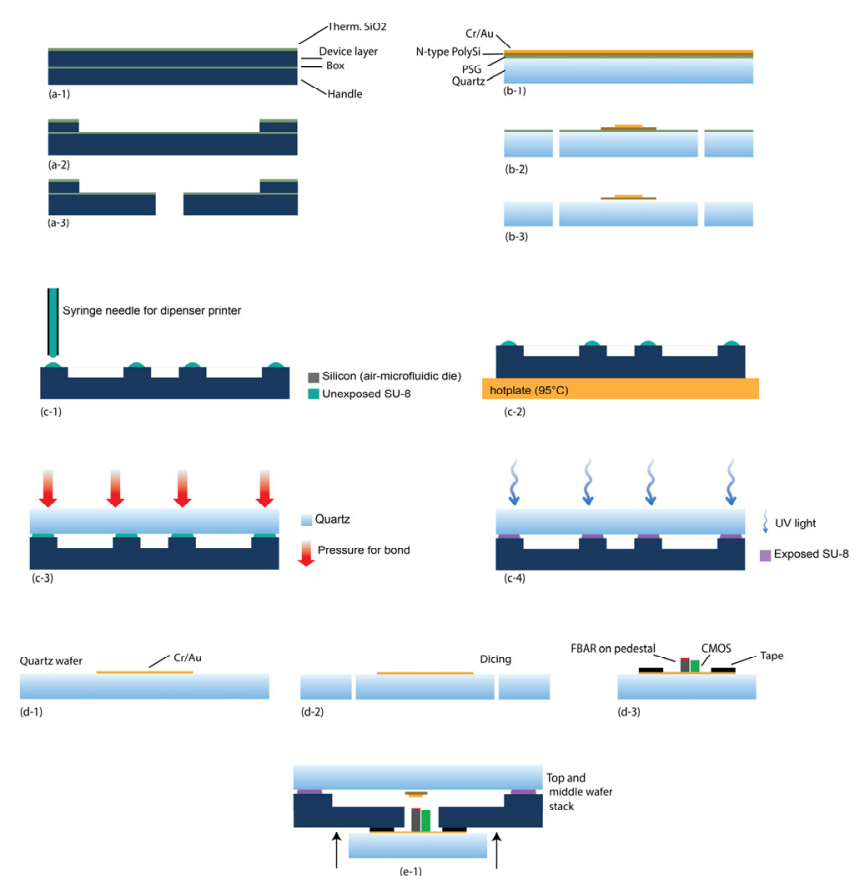

Fig. 4. Fabrication process flow for the middle wafer (a1-3), top wafer (b1-3), dispenser printing of SU-8 and bonding of top and middle wafers (c1-4), bottom wafer fabrication (d1-3), and final assembly (e-1)

\section{Thermophoretic heaters (top wafer)}

The fabrication process for the thermophoretic heaters is illustrated in Fig. 4-b. First, a $1 \mu \mathrm{m}$ thick layer of phosphosilicate glass (PSG) is deposited using low-pressure chemical vapor deposition (LPCVD) process $\left(2: 4: 1 \mathrm{SiH}_{4}, \mathrm{O}_{2}, \mathrm{PH}_{3}\right.$ at $\left.450^{\circ} \mathrm{C}\right)$ to form the sacrificial layer for HF release. A $2 \mu \mathrm{m}$ thick layer of in-situ doped polysilicon is then deposited through a LPCVD process $(1: 1$ $\mathrm{SiH}_{4}, \mathrm{PH}_{3}$ at $615^{\circ} \mathrm{C}$ ) over the PSG layer. To form low resistance connections to the heaters, a $100 \mathrm{~nm}$ thick layer of gold (Au) with a $5 \mathrm{~nm}$ chrome adhesion layer $(\mathrm{Cr})$ is evaporated on top of the polysilicon layer (b-1) and subsequently patterned using a photolithography process and etched using an iodine-based gold etchant and CR-7. To create the resistive heater elements, the polysilicon layer is etched by a reactive ion-etch (RIE) process $(90 \%$ SF6, 10\% O2, $66 \mathrm{sccm})(\mathrm{b}-2)$. The wafer is subsequently diced and the individual die are soaked for $10 \mathrm{~min}$ in $49 \% \mathrm{HF}$ to release the heaters from the substrate (b-3).

\section{Microfluidic channels (middle wafer)}

The fabrication process of the air-microfluidic channels is illustrated in Fig. 4 -a. $1 \mu \mathrm{m}$ thick layer of silicon dioxide is thermally grown on a silicon-on-insulator (SOI) wafer with $200 \mu \mathrm{m}$ device layer (a-1). This layer serves as the hard mask for subsequent deep reactive ion etch (DRIE). Channels are then photo-lithographically patterned and etched through the device layer using a two-step DRIE processes (a-2) (Oxide etch: $\mathrm{C}_{4} \mathrm{~F}_{8} / \mathrm{H}_{2} / \mathrm{He} 15 \mathrm{sccm}$, $8 \mathrm{sccm}$ and 174 sccm at $4 \mathrm{mT}, 1500 \mathrm{~W}$, Bias $350 \mathrm{~W}$ at $13.56 \mathrm{MHz}$. Si etch and passivation: $7 \mathrm{sec} \mathrm{SF}_{6} / \mathrm{O}_{2} 100 \mathrm{sccm} / 13 \mathrm{sccm}$ at $18 \mathrm{mTorr}, 5 \mathrm{sec} \mathrm{C}_{4} \mathrm{~F}_{8}$ $100 \mathrm{sccm}$ at $18 \mathrm{mTorr}$ and $600 \mathrm{~W}, 15 \mathrm{~W}$ bias, $380 \mathrm{KHz}$ ). A two-step backside DRIE etch (handle and BOX) is subsequently performed to open the inlet (i), outlet (ii), and FBAR insertion opening (a-3).

\section{Resonator and circuitry substrate (bottom wafer)}

The bottom wafer fabrication process is illustrated in Fig. 4.(d$1-\mathrm{d}-3$ ). A $5 \mathrm{~nm} / 100 \mathrm{~nm}$ layer of $\mathrm{Cr} / \mathrm{Au}$ is evaporated on a quartz wafer and patterned and etched lithographically to define electrodes and provide connections between electronic components, followed by the sub-dicing of the wafer. A CMOS chip and a $475 \mu \mathrm{m} \times 1 \mathrm{~mm}$ $\times 1 \mathrm{~mm}$ silicon pedestals are die attached to the board and FBAR is attached on top of the pedestal by a non-conductive epoxy followed by a $60 \mathrm{~min}$. curing at $150{ }^{\circ} \mathrm{C}$. The FBAR is then wire-bonded to the CMOS and CMOS is wire-bonded to the gold electrodes. Then an $80 \mu \mathrm{m}$ thick adhesive polymeric seal is attached around the perimeter of the FBAR and CMOS to provide a seal for the opening through the middle wafer. Assembling the bottom board to the stack of the middle and top wafer is illustrated in Fig. 4.(e-1). The thickness of the seal also sets the height that the FBAR penetrates into the microfluidic channel. Finally, bypass capacitors and RF cables are soldered to provide off-board power and RF connections.

\section{Wafer bonding}

Bonding of the air microfluidics wafer (middle wafer) to the quartz wafer containing the released microfabricated gold heaters (top wafer) is a critical part in the assembly of PM sensor. Perfect sealing of microfluidic channels is necessary because even a small air leak can affect the flow rate in the minor and major channels and impair the functionality of the sensor. In this case, there are considerations which limit the use of some common wafer bonding methods such as direct bonding or anodic bonding. Different wafer materials (silicon and quartz), non-conformal geometry, and dielevel bonding, precludes the use of common direct bonding methods. Lack of alkali ions in quartz precludes the use of anodic bonding. In contrast, adhesive bonding which is a popular wafer bonding method for MEMS applications and microfluidics [4] is favored because of its compatibility with different substrate materials and non-planar geometries, ease of application and relatively inexpensive process, and possibility of selective bonding.

SU-8 polymer (negative photoresist) is frequently used in MEMS adhesive bonding applications; however, spin coating of SU-8 on either of the middle or top wafers is not practical because it can fill the microfluidic channels or damage the heaters suspended just beneath the quartz wafer. Thus selective deposition of the SU-8 adhesion layer via a dispenser printer is a better approach to apply SU-8 onto the desired areas. 
Dispenser printing and subsequently the wafer bonding process are schematically displayed in Fig. 4-(c1-c4). SU-8 3025 (Microchem) is dispenser printed using a custom printer on the middle wafer to provide an adhesive seal around the perimeter of the minor and major channels, as well as around the virtual impactor (c1). After printing, the SU-8 is soft baked at $95^{\circ} \mathrm{C}$ for 3 minutes on a hotplate (c2). Then, the top wafer is aligned with the airmicrofluidics wafer and placed on top of it, followed by the application of a constant bonding force (such as adding weights on top) (c3). The SU-8 is then exposed to UV light for 60 seconds through the quartz wafer for polymerization (c4). Following exposure the SU-8 is further cured at $95^{\circ} \mathrm{C}$ for 5 minutes to provide a strong bond. Fig. 5(a-b) shows optical micrographs taken during the SU-8 dispenser printing process, while, Fig. 5(c) shows an image of the middle wafer after the SU-8 pattern is printed but before the bonding.
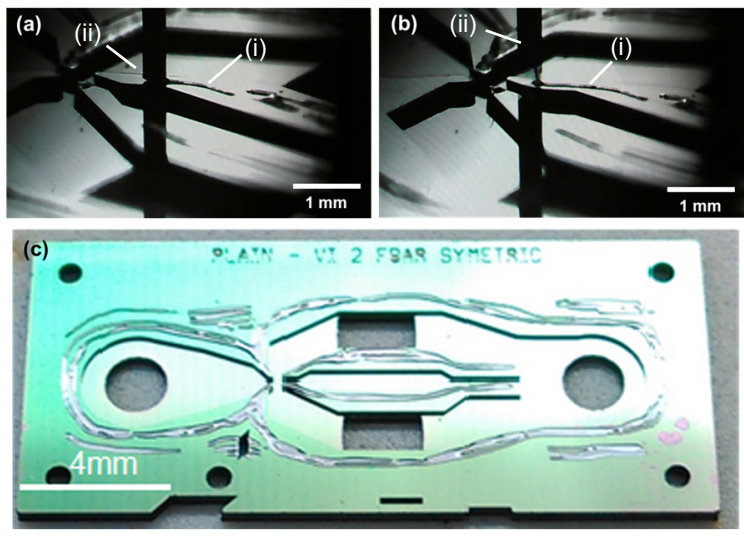

Fig. 5. (a-b) Optical micrographs showing the dispenser printed $S U-8$ (i) around the collection probe of the microfabricated VI. The nozzle of the dispenser printer is visible (ii). (c) Dispenser-printed pattern of SU- 8 prior to bonding with the top wafer.

\section{SU-8 spreading and transformation}

Spreading of the printed SU-8 after applying the top wafer and a given bonding force must be taken into consideration to avoid the adhesive from reaching into microchannels or unwanted areas. Spreading of a liquid droplet placed on a solid surface is a common phenomenon. Under equilibrium, the well-known Young-Laplace equation governs the behavior of a liquid droplet placed on a solid surface and the angle of contact is calculated based on the interface tension between solid-liquid, solid-vapor, and liquid-vapor interfaces.

It has been shown in [5-6] that the area of contact of a droplet with a solid surface under a force will increase with time at first, but then it reaches a saturation value and does not increase significantly beyond that point. By calculating the saturation area we are able to precisely include safety margins in the design of printing pattern map. An inverse configuration space transformation can be applied to convert the desired pattern to the pattern that will be printed by the dispenser printer.

The saturation area of contact of a constant volume of liquid $V_{f}$ with the solid surface under the load of $M(\mathrm{~kg})$ is calculated from (1), which is derived by Nag et al. in [5]:

$$
A_{\text {sat }}^{4}-A_{0}^{4}=\frac{8 \pi v_{\max } V_{f}^{2} M}{3 \mu},
$$

where it is assumed that the load $M$ is dropped and the maximum kinetic energy is dissipated by the viscous droplet as the load plate comes to a rest. Thus the corresponding maximum velocity $v_{\text {max }}$ can be calculated at the point of contact. $A_{0}$ denotes the initial area of the contact while $A_{\text {sat }}$ denotes the saturation area and $\mu$ is the dynamic viscosity of the fluid.

In our case, as the majority of printing patterns of SU-8 are rectangles with very high aspect ratio (very thin and long lines), the spread of the SU-8 along the length is insignificant compared to spread along the width, and can be neglected.

$$
\left(w_{s} l_{s}\right)^{4}-\left(w_{0} l_{0}\right)^{4}=\frac{8 \pi v_{\max } V_{f}^{2} M}{3 \mu}
$$

$l_{s} / l_{0} \approx 1$ (for rectangular patterns with high aspect ratio compared to $\left.w_{s} / w_{0}\right)$,

$$
w_{S}^{4}-w_{0}^{4}=\frac{8 \pi v_{\max } w_{0}^{2} h_{0}^{2} M}{3 \mu l_{0}^{2}}
$$

It should be noted that the constant volume of liquid is obtained from $V_{f}=w_{0} h_{0} l_{0}$ where $w_{0}$ and $h_{0}$ are the initial width and height of the pattern of SU-8. These can be modified by the dispenser printer's specifications such as syringe's tip size and air pressure.

A series of experiments have been designed and carried out to evaluate Eq. (3). Four lines of SU-8 3025 with width of $100 \mu \mathrm{m}$, height of $40 \mu \mathrm{m}$ and length of $5 \mathrm{~mm}$ were printed to form a square shaped frame. Then the top plate (glass with weight of $20 \mathrm{~g}$ ) followed by a load of $0.4 \mathrm{~kg}$ were placed on top. The process was captured using a camera (Canon EOS) at the rate of 30 frames per second and the images were analyzed using Adobe Premiere. The velocity $v_{\max }$ is attained as the load comes to the rest, calculated at $1 \mathrm{~m} / \mathrm{s}$. The area of contact is saturated after $\sim 60$ seconds. Specification of SU-8 3023 from are listed in Tab 1.

Table 1: Specifications of SU-8 3025

\begin{tabular}{|c|c|c|}
\hline $\begin{array}{c}\text { Density } \\
\left(\mathrm{kg} / \mathrm{m}^{3}\right)\end{array}$ & $\begin{array}{c}\text { Kinematic } \\
\text { Viscosity } \\
(\mathrm{cSt})\end{array}$ & $\begin{array}{c}\text { Dynamic } \\
\text { Viscosity } \\
(\mathrm{kg} / \mathrm{ms})\end{array}$ \\
\hline 1143 & 4400 & 50.292 \\
\hline
\end{tabular}

From Eq. (3) the saturation width is calculated to be $128 \mu \mathrm{m}$, which is consistent with the experimental results obtained by the image and video analysis. A $28 \mu \mathrm{m}$ spread (width) was measured from a line $100 \mu \mathrm{m}$ wide, however this does not take into account the spread as a result of unwanted lateral movements of the top wafer during the bonding process.
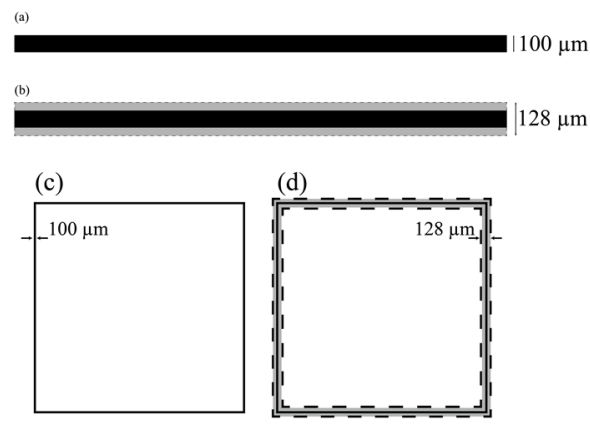

Fig. 6. Inverse configuration space transformation for a high aspect ratio line pattern $(a, b)$ and a square test pattern $(d, c)$.

The idea of the inverse configuration space transformation is displayed Fig. 6. Based on Eq. (3), the desired pattern (gray and black, (b) and (d)) is obtained by printing SU-8 in black areas only ((a) and (c)). 


\section{EXPERIMENTAL RESULTS}

A representative result from exposing the MEMS PM2.5 sensor to smoke is shown in Fig. . The flow through the sensor was controlled using an external pump and the flowrate was set to 6 $\mathrm{mL} / \mathrm{min}$. The experimental results indicate the sensitivity of our sensor at $5 \mathrm{~Hz} \times \mathrm{m}^{3} / \mu \mathrm{g} \times \mathrm{min}$, with a (LOD) of $1 \mu \mathrm{g} / \mathrm{m}^{3}$. The sensor showed a tendency to lose some mass after excessive deposition of smoke on the FBAR surface, likely due to the evaporation of semivolatile constituents of the incense as the smoke equilibrates with ambient air. This is a common feature with continuous measurement methods and can be potentially used for PM speciation.

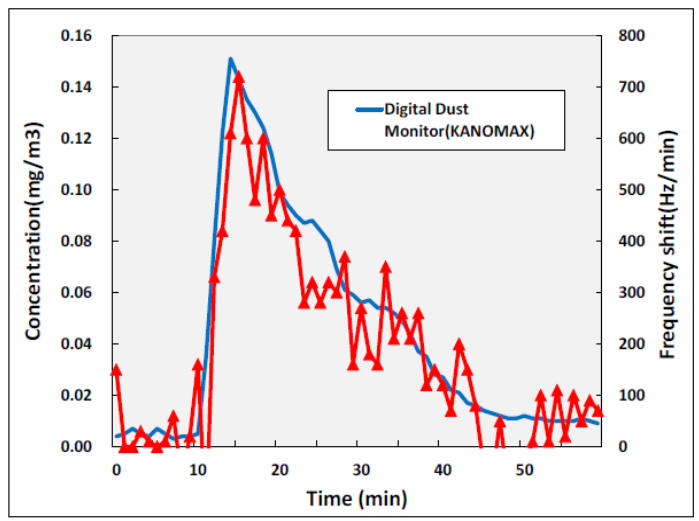

Fig. 7. Experimental results from the exposure of the MEMS PM2.5 to incense smoke in the range of $100-300 \mathrm{~nm}$ AD.

\section{CONCLUSION}

Due to the serious health hazards associated with fine PM (PM2.5), development of personal PM monitors is of great interest. In this work we presented the design, fabrication, and initial testing results of a MEMS air-microfluidics lab-on-a-chip PM2.5 sensor with improved VI design, fabrication process, and novel SU-8 dispenser printing based wafer bonding. The sensor has shown the sensitivity of $5 \mathrm{~Hz} \times \mathrm{m}^{3} / \mu \mathrm{g} \times$ min, with a detection limit of $1 \mu \mathrm{g} / \mathrm{m}^{3}$. This small footprint air-microfluidic lab-on-a-chip presents an initial platform for future more complex air-microfluidic circuits.

\section{ACKNOWLEDGEMENTS}

This work was in part sponsored by grant from Kanomax, contract \#2015-03267. Microfabrication tasks were performed at the Marvell Nanofabrication Laboratory at UCB and Nanofabrication Core Facility at UIC. We would like to acknowledge the support of Rich Ruby and Avago Technologies Inc. for providing high quality FBARs and Kanomax for conducting collaborative testing of the sensors. Finally, we acknowledge the Transducer Research Foundation for providing generous travel support. The U.S. Environmental Protection Agency, through its Office of Research and Development, collaborated in the research described here. It has been subjected to Agency review and approved for publication.

\section{REFERENCES}

[1 ] Doering, F., I. Paprotny, and R.M. White, Technical Digest of the 15th Solid-State Sensor and Actuator Workshop, SC, June 2012, Hilton Head Isl.

[2] Paprotny, I., et al. "Microfabricated air-microfluidic sensor for personal monitoring of airborne particulate matter: Design, fabrication, and experimental results." Sensors and Actuators A: Physical 201 (2013): 506-516.

[3] Shinyei Technology Co Ltd, "Particle Sensor," available online http://www.shinyei.co.jp/stc/optical/main_pm2_e.html downloaded 1/12/2016

[4] Niklaus, F., et al. "Adhesive wafer bonding." Journal of applied physics 99.3 (2006): 031101.

[5] S. Nag, S. Dutta, and S. Tarafdar. "Scaling laws for spreading of a liquid under pressure." Applied Surface Science 256.2 (2009): 353-355

[6] S. Nag, T. Dutta, and S. Tarafdar. "Spreading of fluids on solids under pressure: Slip and stick effects." Journal of colloid and interface science 356.1 (2011): 293-297.

\section{CONTACT}

* I. Paprotny, tel: +1-312-996-1924; paprotny@uic.edu 